\title{
Systems analysis of the issue of ensuring reliable operation of vehicles
}

\author{
Ivan Tikhii ${ }^{1, *}$, and Viktor Kashkovsky ${ }^{1}$ \\ ${ }^{1}$ Irkutsk State Transport University, 664047 Irkutsk, Russia
}

\begin{abstract}
The paper describes relevance of the issue of ensuring reliable operation of vehicles for providing the required level of economic efficiency and safety of transportations via transport systems. Systems analysis method is used for solving the scientific problem. The place of vehicles is defined in the general classification of technical systems on the basis of systems analysis. The study proposes to divide the criteria of operational efficiency in accordance with the groups of properties of technical objects and shows their interrelation. As a result of the study, the generalized structure of integrated complexes of on-board equipment (ICOE) of vehicles is formed. Analysis of ICOE properties and their effect on the complex reliability is performed from the standpoint of systems approach is performed. Theoretical substantiation is provided for the necessity and the possibility of implementing the principles of survivability and fault tolerance of ICOE to ensure their effective use. The application of mutual control structures for the organization of on-board computer system diagnostics as a central part of ICOE is substantiated.
\end{abstract}

\section{Introduction}

Achieving and maintaining the required level of efficiency of transport systems has been and remains an important scientific and practical problem, due to the high importance of technological processes of transportation of goods for the development of the economy. The problem has a complex nature, since operational efficiency of vehicles is defined by multiple factors. For technical systems, reliability represents one of the key factors, for that reason a big part of studies is dedicated to the issues of providing the required efficiency level with regard to reliability and safety criteria.

Methods and techniques of solving this problem were mostly developed by various research and design teams for vehicles of a certain type on the basis of the account of their individual construction peculiarities and properties. At the same time, presently, due to the use of various technologies (including information ones) and cutting-edge design solutions while designing vehicles for different purposes, they gained common properties as systems.

This statement defines the necessity and rationality of using systems analysis method for selecting and substantiating the effective solution to the studied problem based on the adequate presentation of vehicles' properties and application conditions (limitations).

\footnotetext{
*Corresponding author: tiviv@list.ru
} 


\section{Research methods}

First, let us define the object and the subject of study in accordance with the key principles of systems approach; it shall be done by specifying general statements on the studied topic.

Object of study. All objects of engineering activity, (without applying for universality of the given classification), are reduced to the following groups: - technical objects (technical products):

- technical systems;

- technical complexes;

- organizational and technical systems;

- ergatic systems (man-machine systems).

At present, the technical literature, including the normative one, widely uses the concept of technical product, which combines structural features of technical objects, systems and complexes of various types and purposes. The technical system, like any other system, is understood as a set of interrelated elements united by common operational purposes. The term complexes is usually used to refer to complex systems that differ in the number of tasks to be solved for their purpose, the heterogeneity of its elements, a large number of objects, involving their integration into separate subsystems.

Transport systems considered in the study can be classified by organizational characteristic to the so-called large systems having a hierarchical multilevel structure, the components of which at different levels are represented by all of the above types of objects and systems.

In this paper, the transport systems are divided into two components: technical and organizational-methodical, the technical component is chosen for further study. From the technical component of the transport system, the actual vehicles (locomotives, airplanes, cars, etc.) are distinguished, which form a park of similar objects according to the concepts of the theory of state control.

In general, the classification of objects of engineering activity involves their division into two levels: technical objects (TO) and technical systems (TS), depending on the objectives of the study. So, if the knowledge of interval characteristics of the object properties without studying its structure is enough to obtain the result, then it is presented as a TO. Otherwise, it is presented as a vehicle, which is a set of interrelated elements (subsystems). The extent and methods of division,as well as the range of distinguished components are determined not only by the objectives of the study, but also by the methods of their achievement. Therefore, this study uses the general formulation of the object of study as a generalized concept of vehicle.

Subject of study. The concept of efficiency in general and, in particular, the functioning of technical objects can be defined as the degree of performance of functions the object is designed to perform, which depends on the state of TO. The paper [1] proposes and justifies the concept of the state of a technical object, and the paper [2] formulates is as follows: a form of manifestation of its qualitative certainty, characterized by the degree of compliance of the parameters of the properties and characteristics of the system of existence of this object with the established requirements defined in time and space.

The presented definition is adapted to the solution of the problems of TO state management, as it reflects not only the semantic content of the concept, but also outlines the method of assessing this state.

In this definition, the system of existence of technical objects (SETO) is a set of the technical object itself, the associated systems and the environment involved in the process of existence. These characteristics should be divided in accordance with the structure of the SETO into: essential properties of technical objects, related systems, environment and the process of existence. 
For technical objects, a common specification is a complex of interrelated essential properties: constructability, functionality, reliability, safety, efficiency, ergonomics, environmental friendliness. Here: constructability involves characteristics of shape, size, etc., the change of which leads to a change in the state of TO; functionality denotes the number and type of functions performed in accordance with the purpose of TO; reliability is interpreted in accordance with GOST 27.002-89 [3] and the other properties are used in their conventional sense.

This division of the whole complex of essential properties TO into particular properties allows presenting the complex concept of TO effectiveness as the degree of achievement of the operational goals according to the criteria of constructability, functionality, reliability, safety, efficiency, ergonomics and environmental friendliness. By the same criteria it is possible to divide ways and methods of increasing operational efficiency of functioning TO, which in most cases have different effects on the result.

Thus, the main attention of this study is focused on the development of a method to improve the efficiency from the standpoint of the criteria of vehicles reliability and safety. At the same time, taking into account the complexity of the concept of TO effectiveness, the proposed solutions will be also evaluated by other criteria.

To solve the task, let us analyze some properties of vehicles as systems, in accordance with their classification proposed in [4] and developed in [5, 6].

Integrity and openness. The properties of integrity and openness should be correlated with the (large) transport system based on a distinctive feature - transportation of people and goods in different environments (earth, water, air, space).

The internal heterogeneity (the distinctiveness of parts) allows singling out means of transport from a large system into a separate subsystem (a park of similar objects).

Structuredness. In turn, modern vehicles, regardless of their type, are technical systems of varying degrees of complexity, consisting of several subsystems, united by a hierarchical structure. For example, aircraft at the top level of the hierarchy consists of airframe, engine and on-board equipment systems. The on-board equipment includes subsystems, including power supply, control, navigation, radar and communication, life support, etc. Due to the significant difference in the types of subsystems included in the on-board equipment, it is classified as an integrated technical complex. At the same time, the integration of the equipment is carried out on the basis of common computing resources [7, 8]. The typical structure of the aircraft integrated complex of on-board equipment (ICOE) is shown in Figure 1.

The given structure is the implementation of general trends in development of ICOE based on the deep integration of systems and generalization of software and hardware resources. Such structural solutions are driven by the increasing need to increase the functionality of the equipment while seeking to reduce its cost and operating costs. That is, in terms of system analysis, there are increasing requirements to improve the efficiency of on-board equipment on the criteria of functionality and efficiency. The possibility to fulfill these requirements is provided by the state-of-the-art level of technological development and the hardware components of microchip equipment. 


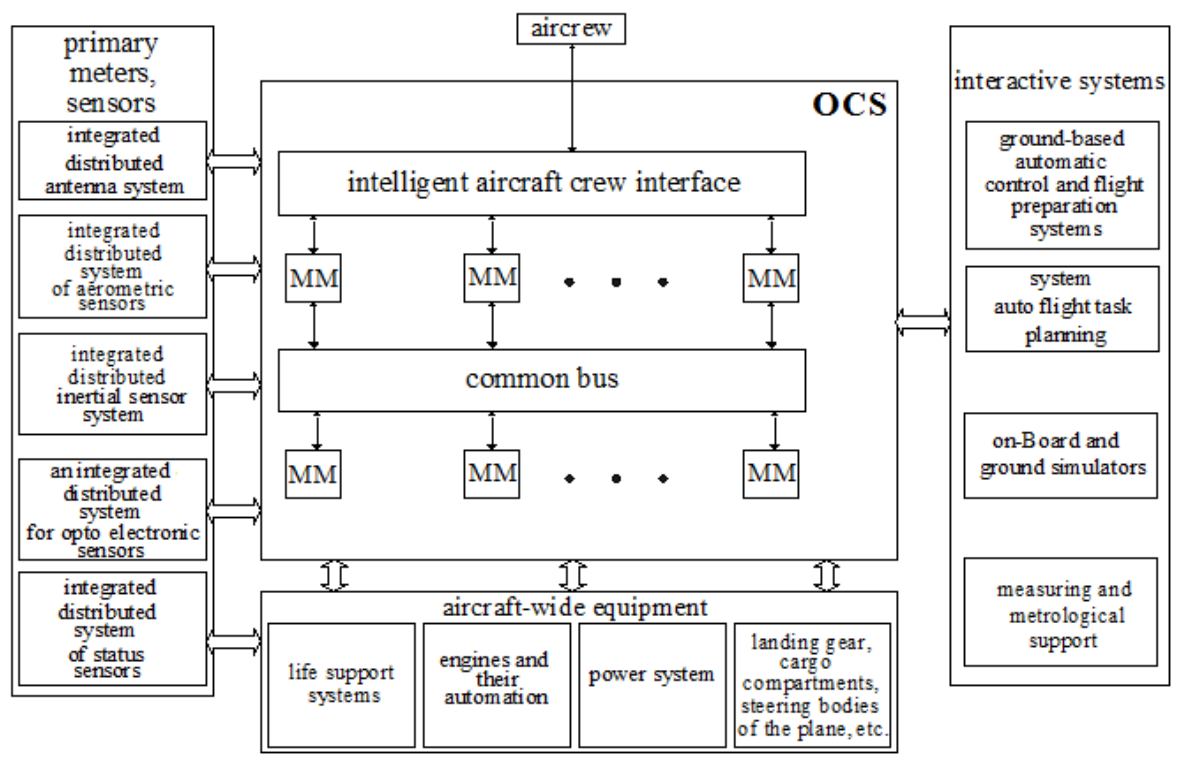

Fig. 1. Typical structure of ICOE.

While exploring the property of structuring, we present a set of on-board equipment consisting of two main parts: the central part, which is the on-board computer system (OCS), and the peripheral, which includes all the rest subsystems specialized to perform individual functions.

The on-board computer system consists of microprocessor modules (MM) and preform functions of overall control, control and transfer of information between the equipment units. According to the architecture, modern multi-component computing systems are distributed, i.e. the control functions are distributed between the modules of the system, and a significant degree of autonomy of the modules is achieved while maintaining a sufficient level of connectivity [9].

Functionality. In studying the properties of ICOE functionality from the standpoint of reliability, it is important to distinguish the functions of individual subsystems based on different degrees of impact on the safety of transport, and for on-board computing systemsthe important function is implementation of self-control of individual modules.

Variability. The variability property of ICOE as a system by the criteria of reliability and safety is primarily connected with stochasticity of the processes of reliability components variation and degradation of the structure caused by these processes.

Emergence. Unification of all computing resources in a distributed microprocessor OCS allows organizing the process of diagnosing the status of system components, additionally to the function of self-control,

As a fundamental property of all systems, inherence characterizes consistency, compatibility, adaptability to the environment and is of particular interest for the studied problem. For artificial systems, which include ICOE, inherence determines the degree (quality) of the performing given functions, that is, the efficiency of the systems.

In accordance with the general principles of the systems approach [7], the process of evaluating effectiveness of the systems can be formally represented by the scheme (Fig. 2). 


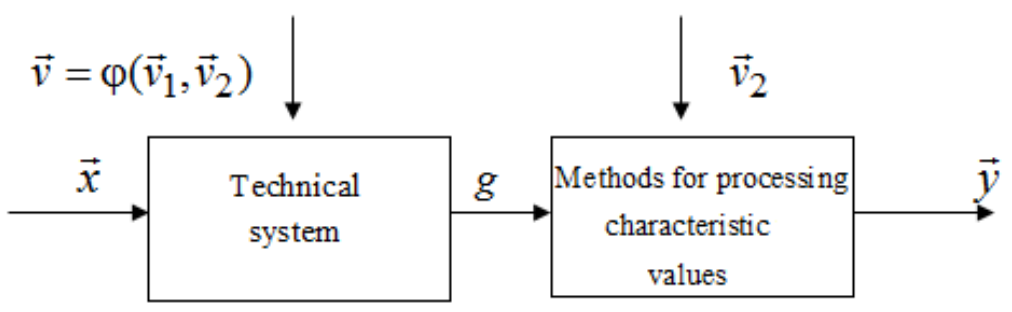

Fig. 2. Experiment as a system.

The analytical process for determining efficiency can be described as $\vec{y}=F\left[\psi\left(\vec{x}, \varphi\left(\vec{v}_{1}, \vec{v}_{2}\right)\right)\right]$, where $\psi$ - the law of the system operation; $F$ - methods of data processing; $\vec{x}$ - the perturbation vector (properties of the system); $\vec{v}=\varphi\left(\vec{v}_{1}, \vec{v}_{2}\right)$ - the control vector; $\vec{v}_{1}, \vec{v}_{2}$ - vectors of controlled and uncontrolled parameters (constraints); $\varphi$ - the function of formation of the law of experiment control; $\vec{y}$ - the vector of the results of the experiment to determine the efficiency.

In this statement of the research problem, the efficiency of the system is determined by finding the vector $\vec{y} \subset A$ in the set area $\mathrm{A}$ and its values (the system becomes fully observable) and is achieved by specifying the vector $\vec{v}, F$ and $\psi$ (Fig. 3.): Conditions for the system observability: for $\vec{y} \subset A$ (A - set range of values):

1. for $\vec{v}_{2}=$ const $\psi=$ const,

2. for $\vec{v}_{2}=$ var, $\psi_{0}$ corresponds to $F_{0} ; \psi_{1}$ corresponds to $F_{1}$;

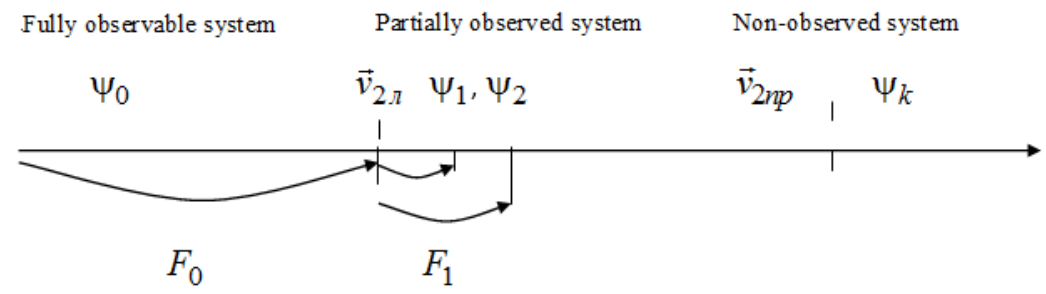

Fig. 3. System monitoring areas.

Under the operating conditions of the system, failure to comply with the $\vec{v}_{2}$ restrictions leads to a change in $\psi$ and thus to the emergence of a new $\psi_{1}$ system, emergent to the original $\psi_{0}$. In this case, the system is shifted to the area of partial observation or becomes unobservable. Therefore, changing the $\psi$ transformation in the law of operation requires an adequate change in the $F$ transformation.

\section{Results}

Thus, the operational efficiency, in accordance with the principles of a systems approach, shall be understood as the degree of adaptability of the ICOE to performing its tasks in compliance with all assumptions and limitations related to operation. With regard to such a complex and multifaceted system as the ICOE, they form a rather extensive set of interrelated conditions (limitations), the meeting which is necessary to achieve the required efficiency. 
In this statement of the problem of efficiency improvement, the solutions techniques can be divided into three groups:

- removal or lowering the level of limitations;

- adaptation of the system to existing limitations;

- a combination of the first two methods.

The choice of methods is determined by the properties of the system under study. Let us show this by the example of the problem to be solved.

Based on the objectives of this study, from the whole set of restrictions only those destabilizing factors are chosen that directly affect the efficiency of the system in terms of reliability and security. In this case, they can be divided into two groups of factors:

- external impact of natural (environmental parameters) and artificial (damaging factors) origin;

- internal impact caused by the mutual negative influence of the system elements.

Ensuring the inherence of ICOE to external destabilizing effects requires giving the complex the properties of survivability, which is especially important for systems used in extreme conditions of emergency situations and military actions. Internal destabilizing factors for ICOE are most often represented by electromagnetic compatibility problems, as well as mechanical effects in the form of shocks, vibrations, pulsations and acoustic noises. In these cases, providing the necessary level of inherence of the complex requires attributing to the complex additional properties of fault tolerance, in addition to the use of highly reliable elements.

To implement the properties of survivability and fault tolerance in ICOE, it is required to provide a solution to two main tasks:

- determination of the type of state (diagnosis) of the system and

- its restoration at the selected level of degradation.

The solution of these problems is carried out by the corresponding subsystems also under limited conditions. Thus, system recovery is based on the existing level of redundancy in the system and the division of system functions by the degree of security impact.

If the system of diagnostics is represented as a set of the object of diagnosis, diagnostic device and the process of diagnosis, the main limitation in the system operating under the influence of destabilizing factors, will be a high probability of failure (damage) of the diagnostic device.

This limitation can be removed by organizing the mutual control structures (MCS) in multiprocessor computing systems, which are the central part of the ICOE (the method was proposed and developed in $[10,11,12,13,14])$. The method involves determination of the technical state of OCS by the results of mutual test checks of each other's state performed by the operating modules of the system (the module is understood as a separate computer), at the same time it remains possible to obtain a reliable diagnosis even in the presence of the failed modules in the structures of mutual control.

When using the ICS method, it is necessary to take into account the limitations on the diagnostic model used, which suggest a high probability of non-constant outflow situations. These situations are caused by failures, intermittent failures, incompleteness of checking tests, communication failures, changes in the outflow situation during the diagnostic cycle. The most complete account of these limitations is provided by the authors of model proposed in the study "On diagnosing outflow situations of various types in computing systems" [15].

\section{Conclusion}

Thus, the study analyses the properties of the ICOE as a system functioning under the influence of external and internal destabilizing factors (limitations), with the use of systems approach. Based on the identified properties of the system, the direction for solving the problem of ensuring the effective operation of ICOE vehicles based on the criteria of reliability and safety is justified. This direction provides attributing the properties of fault tolerance and survivability to the system, along with the use of highly reliable elements. At the same time, the degree of implementation of these properties is 
ensured by the use of mutual control structures and is set based on the required performance indicators of a particular vehicle.

The obtained results are unique and can be applied for ensuring fault tolerance of integrated technical complexes and systems of various type and purpose.

\section{References}

1. I.I. Tikhii, V.V. Kashkovsky, Modern Technologies. System Analysis. Modeling, Vol. 4 (48), 134 - 139 (2015). (in Russian)

2. I.I. Tikhii, V.V. Kashkovsky, L.V. Arshinsky, FJEC, Vol. 103, Issue 7, 1103 - 1113 (2018).

3. GOST 27.002 - 2015. Dependability in technics. Terms and definitions (Standartinform, Moscow, 2016). (in Russian)

4. F.P. Tarasenko, Prikladnoj sistemnyj analiz [Applied System Analysis] (KNORUS, Moscow, 2010). (in Russian)

5. V.N. Kozlov, Sistemnyj analiz, optimizacija i prinjatie reshenij [System analysis, optimization and decision making] (Prospekt, Moscow, 2016). (in Russian)

6. V.N. Volkova, Sistemnyj analiz informacionnyh kompleksov [System analysis of information systems] (Lan', St. Petersburg, 2016). (in Russian)

7. E.A. Fedosov, V.I. Kasyanchuk, Radioelectronic technologies, 1, 66-71 (2015). (in Russian)

8. I.M. Saidumarov, A.A. Umarov, R.G. Zakirov, Tehnika. Tehnologii. Inzhenerija [Equipment. Technologies. Engineering], 1, 20-24 (2016). (in Russian)

9. S.G. Bobkov, Vysokoproizvoditel'nye vychislitel'nye sistemy [High Performance Computing Systems] (НИИСИ РАН, Москва, 2014). (in Russian)

10. F.P. Preparata, G. Metze, R.T. Chien, IEEE Trans. EC, Vol. 16, 6, 848-854 (1967).

11. F. Barsi, F. Crandoni, P. Maestrini, IEEE Trans.EC, Vol. 25, 6, 585-593 (1976).

12. I.G. Kuhl, S.M. Reddy, IEEE Trans. Comput., 12. 1012-1024 (1984).

13. P.P. Parhomenko, Automation and Remote Control, 5, 126-135. (2009). (in Russian)

14. V.A. Vedeshenkov, Control Sciences, 6, 55-60 (2016). (in Russian)

15. I.I. Tikhii, V.V. Kashkovsky, Instruments and Systems: Monitoring, Control, and Diagnostics, 11, 35-42 (2009). (in Russian) 